\title{
A120 INCREASED IL-22 EXPRESSION BY SYNOVIAL TH17 CELLS DURING LATE STAGES OF ARTHRITIS IS CONTROLLED BY IL-1 AND ENHANCES BONE DEGRADATION
}

Renoud J Marijnissen, ${ }^{1}$ Marije I Koenders, ${ }^{1}$ Ruben L Smeets, ${ }^{2}$ Mark HT Stappers, ${ }^{1,3}$ Cheryl Nickerson-Nutter, ${ }^{4}$ Leo AB Joosten, ${ }^{3}$ Annemieke MH Boots, ${ }^{2,5}$ Wim B van den Berg' '1 Experimental Rheumatology and Advanced Therapeutics, Department of Rheumatology, Radboud University Nijmegen Medical Centre, Nijmegen, The Netherlands; '2Department of Immune Therapeutics, Merck Research Laboratories, MSD, Oss, The Netherlands; ${ }^{3}$ Department of Medicine and Nijmegen Institute for Infection, Inflammation and Immunity (N4i), Radboud University Nijmegen Medical Centre, Nijmegen, The Netherlands; ${ }^{4}$ Pfizer, Cambridge, Massachusetts, USA; ${ }^{5}$ Department of Rheumatology and Clinical Immunology, University Medical Centre Groningen, University of Groningen, The Netherlands

\subsection{6/ard.2010.148981.23}

Background Interleukin (IL)-22 is commonly regarded as a mediator in antimicrobial responses as well as a mediator in inflammatory autoimmune diseases. Although IL-22 and IL-22R have been identified in the inflamed synovium of rheumatoid arthritis patients, the source of IL-22 and contribution to disease pathogenicity remains to be established.

Material and methods The production of IL-22 by T helper cells was assessed. Naive murine $T$ cells were cultured in vitro under Th1, Th2, iTreg and Th17 polarising conditions. Cytokines were measured in the culture supernatants, and effect on differentiation was analysed by flowcytometry. In addition, the in vivo role of IL-22 was investigated in the spontaneous arthritic IL-1Ra-/- mice. Inflamed ankle synovia were isolated and mRNA levels of IL-22 and IL-22R were quantified. IL-1Ra-/- mice were treated with neutralising anti-IL-22 antibodies. Furthermore, synovial cells were isolated and sorted into (CD3, CD19 and CD11b) fractions and analysed for cytokine production.

Results In vitro tests showed that Th17 differentiated T cells produce high IL-22 levels after IL-1 and IL-23 stimulation. Interestingly, the authors found a synergistic increase in the IL-22 production after combining IL-1 and IL-23. During long-term cultures, this synergy of IL-1 and IL-23 on the IL-2 2 production remained, but the balance shifted towards a more prominent role for IL-23. In vivo, IL-17 was upregulated in mildly and severely inflamed tissue, while IL-22 and IL-22R were merely upregulated in severely inflamed synovia. Anti-IL-22 treatment of IL-1Ra-/- mice significantly 
reduced inflammation and bone erosion, suggesting an important role of IL-22 in IL-1-driven joint destruction. Isolating single cells from the inflamed synovia revealed that IL-22 was mainly produced by $\mathrm{T}$ cells. Furthermore, most IL-22 positive $T$ cells co-expressed IL-17 and were increased in the severely inflamed. The cellular data confirm the involvement of Th17 cells in early and late phases of arthritis whereas the involvement of IL-22 by Th17 cells becomes manifest in the later phase of arthritis.

Conclusions IL-22 expression is elevated during early phase of Th17 differentiation caused by IL-1 or IL-23 alone and in synergy. Moreover, a temporal role of IL-22 induction was found for both cytokines. Neutralising IL-22 during experimental arthritis protects against severe joint pathology, revealing an important role for IL-22 during chronic synovial inflammation. IL $-17^{+} \mathrm{IL}-22^{+} \mathrm{T}$ cells were found to be te main IL-22 producers. 